\title{
SIMILARITY SOLUTION OF STAGNATION-POINT FLOW AND HEAT TRANSFER OF A MICROPOLAR FLUID TOWARDS A HORIZONTAL PERMEABLE EXPONENTIALLY ELONGATING SHEET WITH RADIATION, HEAT PRODUCTION/ IMMERSION
}

\author{
R. PARTHIBAN \\ Department of Mathematics, Government Arts College for Men, \\ Affiliated to University of Madras, Chennai, INDIA \\ G. PALANI* \\ Department of Mathematics, Dr. Ambedkar Govt. Arts College, \\ Affiliated to University of Madras, Chennai, INDIA \\ E-mail: gpalani32@yahoo.co.in
}

\begin{abstract}
The current study aims to explore stagnation spot flow of a micropolar fluid about a plain linear exponentially expanding penetrable surface in the incidence of radiation and in-house heat production/immersion. Through similarity mapping, the mathematical modeling statements are transformed to ODE's and numerical results are found by shooting techniques. The impact of varying physical constants on momentum, micro-rotation and temperature is demonstrated through graphs. The computed measures including shear, couple stress, mass transfer and the local surface heat flux with distinct measures of factors involved in this proposed problem are presented through a table.
\end{abstract}

Key words: exponentially stretching sheet, heat spring/drop, micropolar fluid, radiation, suction/injection.

\section{Introduction}

Eringen's [1] micropolar fluids are non-Newtonian fluids which consist of randomly oriented particles exhibiting micro inertial phenomena that affect the undercurrents of the fluid flow. Suspension fluids, muddy fluids and biological fluids such as human blood are some examples of micropolar fluids. Eringen's theory can be applied to the investigation of micro scale fluids and in the exploration of non-Newtonian fluid mechanics.

The investigation of fluid flow over stretching sheets has been a popular research problem for the past few decades (see Mukhopadhyay [2]). The lateral velocity caused by the stretching surface disturbs the adjacent fluid, and the surface convective cooling (Mukhopadhyay et al. [3] and Mahapatra et al. [4]). Such studies have got many interesting industrial applications in processes such as the extrusion of plastic sheets from a die (see Mukhopadhyay and Gorla [5]), the manufacturing of glass by means of blowing, and the cooling of metal plates in a big tub. Uninterrupted casting and spiralling of fibers are some more examples of flow involving elongating surface. Crane [6] obtained an analytical solution of an incompressible viscous flow over an elastic sheet moving with varying velocity. Heat transfer problem over an elongating surface with varying temperature levels was studied by Carragher and Crane [7], Gupta and Gupta [8] and Chakrabarti and Gupta [9]. An analytical solution of heat transfer problem of a micropolar fluid above a non-isothermal penetrable elongating plane was studied by Hady [10]. Hady's problem including suction and injection was investigated using numerical methods by Hassanien and Gorla [11]. An analytical resolution of mixed convective current of micro-polar fluid on a nonlinear elongating plane sheet was given by Hayat et al. [12]. The heat transfer

\footnotetext{
${ }^{*}$ To whom correspondence should be addressed
} 
features along an elongating sheet with suction or blowing and having variable sheet temperature were studied by Chen and Char [13]. Crane's [6] investigation was broadened for various aspects of heat transfer for a linearly stretched surface by Dutta et al. [14], Vajravelu [15], Mukhopadhyay et al. [16], Ishak et al. [17]. The above problems were extended for non linearly stretching sheets and the case for a broader nonlinear powerlaw stretching sheet was analysed by Van Gorder and Vajravelu [18]. They established a unique solution along with some numerical results. Van Gorder and Vajravelu's problem with slip at the boundary was analysed by Mukhopadhyay [19]. The heat transfer features in an electrically conducting fluid above an elongating plane with variable temperature and heat production or immersion were analysed by Vajravelu and Rollins [20]. The mixed convective slip flow of a micro-polar fluid on a hot elongating surface having a constant magnetic flux and heat production/immersion was investigated by Mahmoud and Waheed [21].

The transport properties in the boundary layer region of an exponential elongating sheet were established by Magyari and Keller [22] and for an exponentially porous elongating surface by Elbashbeshy [23]. The thermal radiation influence in the boundary layer region on an exponentially elongating surface was analysed by Sajid and Hayat [24] using HAM. A numerical study of the thermal radiation influence in the boundary layer region on an exponentially elongating sheet investigated by Bidin and Nazar [25]. The mixed convective flow on an exponentially elongating surface having magnetic force field influence was explored by Pal [26]. The magnetic effect on flow properties on an exponentially elongating surface was investigated by Ishak [27].

In a stagnation spot flow, there is a spot on the surface in the flow domain with adjacent velocity being zero and having highest pressure as well as heat transfer. The stagnation spot flow has applications in production processes such as extracting polymers, production of paper, production of glass fiber and many others. The exact solution of the stagnation spot flow on a firm flat plane as well as an axisymmetric plane was analysed by Hiemenz and Homann and these results can be found in the book "Boundary Layer Theory" by Schlichting [28]. The equivalent temperature dispersal problem was analysed by Goldstein [29]. The stagnation spot flow on an axially-symmetric plane for power law liquids was explored by Maiti [30]. The stagnation spot flow for power law liquids of non-Newtonian type was investigated by Koneru and Manohar [31]. Heat transfer in the stagnation spot flow near an elongating sheet was explored by Mahapatra and Gupta [32]. The viscous flow nearby a stagnation spot with the external flow having constant vorticity was analysed by Stuart [33]. Stagnation spot flow near an elongating plane for a micropolar fluid was analysed by Nazar et al. [34].

The radiation heat transfer properties on diverse flows are significant in space engineering and high temperature techniques such as polymer processing, high temperature plasmas, chilling of nuclear reactors and metal fluids. The mass and heat transfer study on the boundary-layer flow of a micropolar fluid over a nonlinearly elongating sheet having radiation effect was studied by Kai-Long Hsiao [35]. The influence of thermal radiation on an MHD stagnation spot flow near an exponentially elongating sheet along with given surface temperature and heat flux was investigated by Sayed Qasim Alavi et al. [36]. The influence of partial slip in the study of the MHD boundary-layer flow past an exponentially elongating sheet having suction/injection as well as thermal radiation was explored by Swati Mukhopadhyay [37]. The impact of thermal radiation on an electrically conducting flow about an exponentially elongating surface with a constant magnetic field by using Rosseland approximation was analysed by Santosh Chaudhary et al [38].

Inspired by the above works, the authors have investigated a stagnation spot flow of an incompressible micropolar fluid on an exponentially elongating penetrable plane in the incidence of thermal radiation with inner heat production/immersion. Through similarity mapping, the mathematical modeling statements are transformed to ODE's and numerical results are found by shooting techniques with Runge-Kutta algorithm.

\section{Details of the flow problem}

The model is associated with a 2-D steady stagnation spot micropolar fluid flow about a plain linear exponentially elongating penetrable surface in the incidence of thermal radiation and in-house heat production/immersion. The coordinate system of the flow is explained in Fig.1. The plane velocity is presumed to be $\hat{u}_{w}(\hat{x})=\hat{U}_{1} \exp (\hat{x} / L)$ and the exterior flow velocity is $\hat{u}_{e}(\hat{x})=\hat{U}_{0} \exp (\hat{x} / L)$ where $\hat{U}_{0}>0, \hat{U}_{1}>0$ are 
constants. Here $\hat{x}$ is the coordinate taken along the flat surface. $\hat{T}_{\infty}$ is the free stream temperature. We also assume the temperature of the stretching sheet to be

$$
\hat{T}=\hat{T}_{w}(x)=\hat{T}_{\infty}+T_{0} \exp (\operatorname{ax} / 2 L)
$$

where $a$ and $T_{0}$ (positive constants) are parameters of temperature distribution on the stretching surface. The impacts of microstructure, viscous dispersion are presumed to be naught and also reduce the total spin $\hat{N}$ to microrotation.

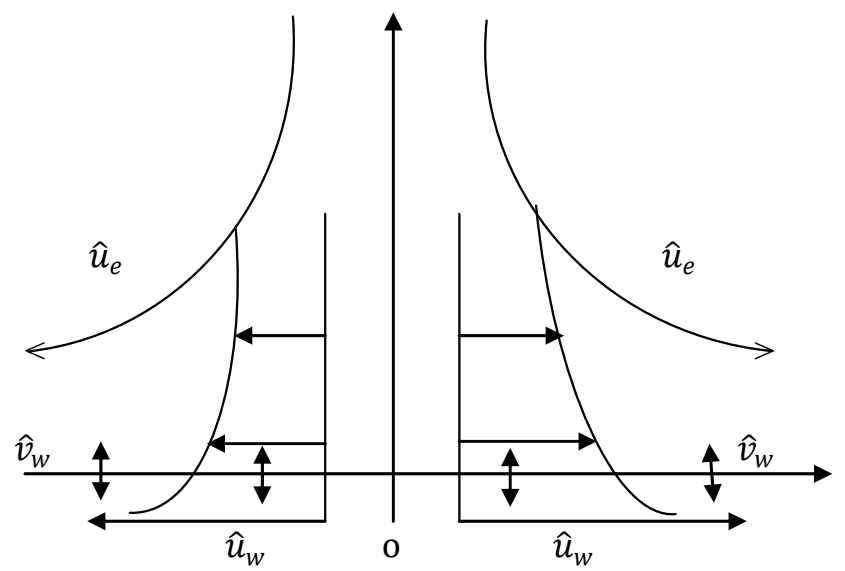

Fig.1. Flow model.

With these assumptions, the continuity, velocity, micro-rotation, as well as energy equations are as follows:

$$
\begin{aligned}
& \frac{\partial \hat{u}}{\partial \hat{x}}+\frac{\partial \hat{v}}{\partial \hat{y}}=0, \\
& \hat{u} \frac{\partial \hat{u}}{\partial \hat{x}}+\hat{v} \frac{\partial \hat{u}}{\partial \hat{y}}=\hat{u}_{e} \frac{\partial \hat{u}_{e}}{\partial \hat{x}}+\left[\frac{\mu+\kappa}{\rho}\right] \frac{\partial^{2} \hat{u}}{\partial \hat{y}^{2}}+\frac{\kappa}{\rho} \frac{\partial \hat{N}}{\partial \hat{y}}, \\
& \rho j\left[\hat{u} \frac{\partial \hat{N}}{\partial \hat{x}}+\hat{v} \frac{\partial \hat{N}}{\partial \hat{y}}\right]=\gamma \frac{\partial^{2} \hat{N}}{\partial \hat{y}^{2}}-\kappa\left[2 \hat{N}+\frac{\partial \hat{u}}{\partial \hat{y}}\right], \\
& \hat{u} \frac{\partial \hat{T}}{\partial \hat{x}}+\hat{v} \frac{\partial \hat{T}}{\partial \hat{y}}=\frac{k}{\rho C_{p}} \frac{\partial^{2} \hat{T}}{\partial \hat{y}^{2}}-\frac{1}{\rho C_{p}} \frac{\partial q_{r}}{\partial \hat{y}}+\frac{Q_{0}}{\rho C_{p}}\left[\hat{T}-\hat{T}_{\infty}\right], \\
& \gamma=\left[\mu+\frac{\kappa}{2}\right] l=\mu\left[1+\frac{\Delta}{2}\right] l
\end{aligned}
$$

where $\hat{u}, \hat{v}$ represent velocity elements in the $\hat{x}, \hat{y}$ - directions respectively; $\kappa$ - vortex viscosity; $\hat{N}$ micro-rotation; $j$ - micro-inertia density; $Q_{0}$ - volumetric heat generation / absorption; $q_{r}$ - radiative heat 
flux; $\gamma-$ spin gradient viscosity; $\Delta=\kappa / \mu-$ micropolar factor and $l=v / \hat{U}_{l}-$ reference length. The Rosseland approximation for radiation is

$$
q_{r}=-\frac{4 \sigma^{*}}{3 k^{*}} \frac{\partial \hat{T}^{4}}{\partial \hat{y}}
$$

where $\sigma^{*}$ is the Stefan-Boltzman constant, $k^{*}$ is the absorption coefficient.

Assuming that the temperature variance inside the flow is such that $\hat{T}^{4}$ can be expressed as a Taylor series around $\hat{T}_{\infty}$, and neglecting upper orders we get $\hat{T}^{4}=4 \hat{T}_{\infty}^{3} \hat{T}-3 \hat{T}_{\infty}^{4}$. Then (2.4) is written as

$$
\hat{u} \frac{\partial \hat{T}}{\partial \hat{x}}+\hat{v} \frac{\partial \hat{T}}{\partial \hat{y}}=\frac{k}{\rho C_{p}} \frac{\partial^{2} \hat{T}}{\partial \hat{y}^{2}}+\frac{16 \sigma^{*} \hat{T}_{\infty}^{3}}{3 \rho C_{p} k^{*}} \frac{\partial^{2} \hat{T}}{\partial \hat{y}^{2}}+\frac{Q_{0}}{\rho C_{p}}\left[\hat{T}-\hat{T}_{\infty}\right] .
$$

Related boundary settings are as follows:

$$
\begin{aligned}
& \hat{u}=\hat{u}_{w}(\hat{x})=\hat{U}_{l} \exp (\hat{x} / L), \quad \hat{v}=\hat{v}_{w}(\hat{x}), \quad \hat{N}=-n \frac{\partial \hat{u}}{\partial \hat{y}}, \quad \hat{T}=\hat{T}_{w}(x) \quad \text { at } \quad \hat{y}=0, \\
& \hat{u}=\hat{u}_{e}(\hat{x})=\hat{U}_{0} \exp (\hat{x} / L), \quad \hat{N} \rightarrow 0, \quad \hat{T} \rightarrow \hat{T}_{\infty} \quad \text { as } \quad \hat{y} \rightarrow \infty .
\end{aligned}
$$

Here $L$-length, $\hat{v}_{w}(\hat{x})$ - transpiration velocity at the wall, $n(0 \leq n \leq 1)-$ boundary value factor. When $n=0$, i.e. $\hat{N}=0$ we have that the non-spiral state. (The micro-components in the concentrated flow particles could not rotate near the wall) and $n=\frac{1}{2}$ represents the disappearing anti-symmetric part of the stress tensor and this implies a feeble concentration. Also, $n=1$ exhibits the turbulent flows. We take the transpiration velocity at the wall as

$$
\hat{v}=\hat{v}_{w}(\hat{x})=-\sqrt{\frac{\hat{U}_{1} v}{2 L}} \exp \left[\frac{\hat{x}}{2 L}\right] s
$$

where $s>0$ refers to the mass suction and $s<0$ refers to the mass injection.

We have the following similarity variables:

$$
\begin{aligned}
& \eta=\sqrt{\frac{\hat{U}_{1}}{2 v L}} \exp \left[\frac{\hat{x}}{2 L}\right] \hat{y}, \quad \psi=\sqrt{2 v L \hat{U}_{1}} \exp \left[\frac{\hat{x}}{2 L}\right] \mathrm{f}(\eta), \\
& \hat{N}=\sqrt{\frac{\hat{U}_{1}^{3}}{2 v L}} \exp \left[\frac{3 \hat{x}}{2 L}\right] \mathrm{g}(\eta), \quad \theta(\eta)=\frac{\hat{T}-\hat{T}_{\infty}}{\hat{T}_{w}-\hat{T}_{\infty}}
\end{aligned}
$$

Equations (2.1)-(2.5) now reduce to:

$$
[1+\Delta] \mathrm{f}^{\prime \prime}+\mathrm{ff} \mathrm{f}^{\prime \prime}-2 \mathrm{f}^{\prime 2}+\Delta \mathrm{g}^{\prime}+2 \varepsilon^{2}=0,
$$




$$
\begin{aligned}
& \lambda g^{\prime \prime}+\mathrm{f} g^{\prime}-3 \mathrm{f}^{\prime} \mathrm{g}-2 B \Delta[2 \mathrm{~g}+\mathrm{f} "]=0, \\
& {[1+R] \theta^{\prime \prime}-\operatorname{Pr}\left[a \theta \mathrm{f}^{\prime}-\mathrm{f} \theta^{\prime}\right]+\operatorname{Pr} Q \theta=0,}
\end{aligned}
$$

Conditions (2.7) and (2.8) become

$$
\begin{aligned}
& \mathrm{f}(0)=s, \mathrm{f}^{\prime}(0)=1, \quad \mathrm{~g}(0)=-n \mathrm{f}^{\prime \prime}(0), \quad \theta(0)=1 \quad \text { at } \quad \eta=0, \\
& \mathrm{f}^{\prime}(\eta) \rightarrow \varepsilon=\frac{\hat{U}_{0}}{\hat{U}_{1}}, \quad \mathrm{~g}(\eta) \rightarrow 0, \quad \theta(\eta) \rightarrow 0 \quad \text { as } \quad \eta \rightarrow \infty .
\end{aligned}
$$

Here $s$ is suction/injection factor, $\operatorname{Pr}=\frac{\mu C_{p}}{k}-$ Prandtl number, $Q=\frac{2 L Q_{0}}{\hat{U}_{w} \rho C_{p}}-$ heat increase/drop factor, $\varepsilon=\frac{\hat{U}_{0}}{\hat{U}_{1}}-$ velocity proportion factor, $R=\frac{16 \sigma^{*} \hat{T}_{\infty}}{3 k k^{*}}-$ radiation factor and $\lambda=\frac{v}{\mu j}$ and $B=\frac{v L}{j \hat{U}_{w}}$ are dimensionless constants.

The physical factors shearing stress $C_{\mathrm{f}}$; the Nusselt number $N u_{\hat{x}}$ are given as:

$$
C_{\mathrm{f}}=\frac{2 \tau_{w}}{\rho \hat{u}_{w}^{2}}, \quad N \hat{u}_{\hat{x}}=\frac{\hat{x} q_{w}}{k\left[\hat{T}_{\infty}-\hat{T}_{m}\right]} .
$$

The surface shear stress $\tau_{w}$; surface heat $q_{w}$ are given by

$$
\tau_{w}=\left[[\mu+\kappa] \frac{\partial \hat{u}}{\partial \hat{y}}+\kappa \hat{N}\right]_{\hat{y}=0}, \quad q_{w}=\left[-k \frac{\partial \hat{T}}{\partial \hat{y}}\right]_{\hat{y}=0} .
$$

From Eq.(2.16) we get

$$
\operatorname{Re}_{\hat{x}}^{1 / 2} C_{\mathrm{f}}=\sqrt{2}[1+(1-n) \Delta] \mathrm{f}^{\prime \prime}(0), \quad \operatorname{Re}_{\hat{x}}^{-1 / 2} N u_{\hat{x}}=-\sqrt{\frac{\hat{x}}{2 L}} \theta^{\prime}(0)
$$

where $\operatorname{Re}_{\hat{x}}=\hat{u}_{e}(\hat{x}) \hat{x} / v$ is the local Reynolds number.

\section{Numerical procedure for solution}

With the shooting method we work out the Eqs (2.10)-(2.12) together with the boundary settings (2.13) and (2.14). In this process, we get the first-order system.

$$
\begin{aligned}
& \mathrm{f}^{\prime}=p, \quad p^{\prime}=q \quad \text { and } \quad q^{\prime}=[1 /(1+\Delta)]\left[-\mathrm{f} q+2 p^{2}-\Delta h-2 \varepsilon^{2}\right], \\
& \mathrm{g}^{\prime}=h \quad \text { and } \quad h^{\prime}=[1 / \lambda][-\mathrm{f} h+3 p g+2 B \Delta(2 g+q)],
\end{aligned}
$$




$$
\theta^{\prime}=z \quad \text { and } \quad z^{\prime}=[\operatorname{Pr} /(1+R)]\left[\operatorname{Pr}\left(a \theta \mathrm{f}^{\prime}-\mathrm{f} \theta^{\prime}\right)+\operatorname{Pr} Q \theta\right]
$$

and the boundary settings

$$
\mathrm{f}(0)=s, \quad p(0)=1, \quad \mathrm{~g}(0)=-n q(0), \quad \theta(0)=1 .
$$

A shooting technique along with Runge-Kutta fourth-order method was employed to solve a group of seven first order differential equations (2.18) to (2.20). To solve them as an IVP, in addition to the four initial conditions (2.21) we need three initial values $q(0), h(0)$ and $z(0)$ i.e., f" $(0), g^{\prime}(0)$ and $\theta^{\prime}(0)$ which are unknown. So initial estimates for $\mathrm{f}^{\prime \prime}(0), \mathrm{g}^{\prime}(0)$ and $\theta^{\prime}(0)$ are used to find a numerical result. For the computation purpose it is compulsory to select an appropriate finite value, say $\eta_{\infty}$ for $\eta \rightarrow \infty$ which guaranties the convergence. The worked out values of $\mathrm{f}^{\prime}(0), g(\theta)$ and $\theta(0)$ at $\eta_{\infty}$ are equated with the required values at $=\eta_{\infty}$. If there is a lack of accuracy in the numerical result, then the guess values $f$ " $(0), \mathrm{g}^{\prime}(0)$ and $\theta^{\prime}(0)$ are revised to proceed as above to find better output results. The process is continued until desired results are obtained.

\section{Results and discussion}

The impact of $\Delta$ - micropolar factor, $\varepsilon=\hat{U}_{0} / \hat{U}_{1}$ - velocity proportion factor and $s$ - suction /injection factor on the boundary layer velocity is shown in Figs 2-5.

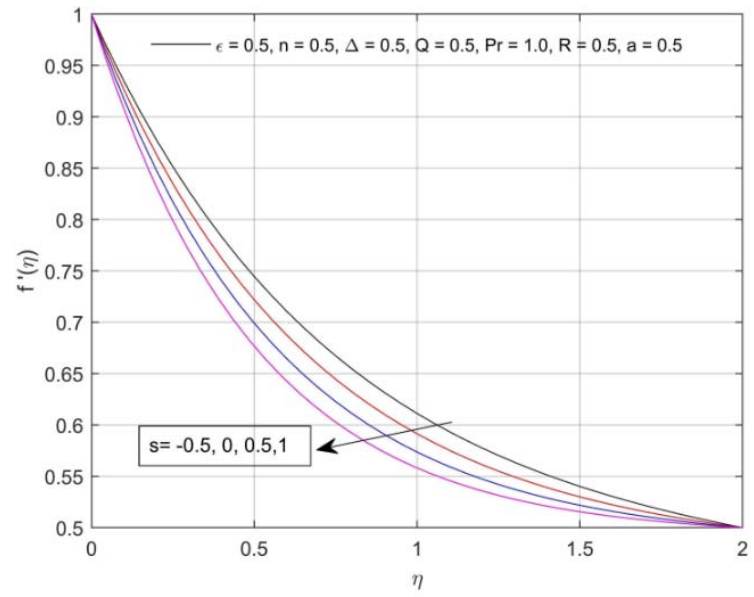

Fig.2. Result for $s$ with $f^{\prime}(\eta)$.

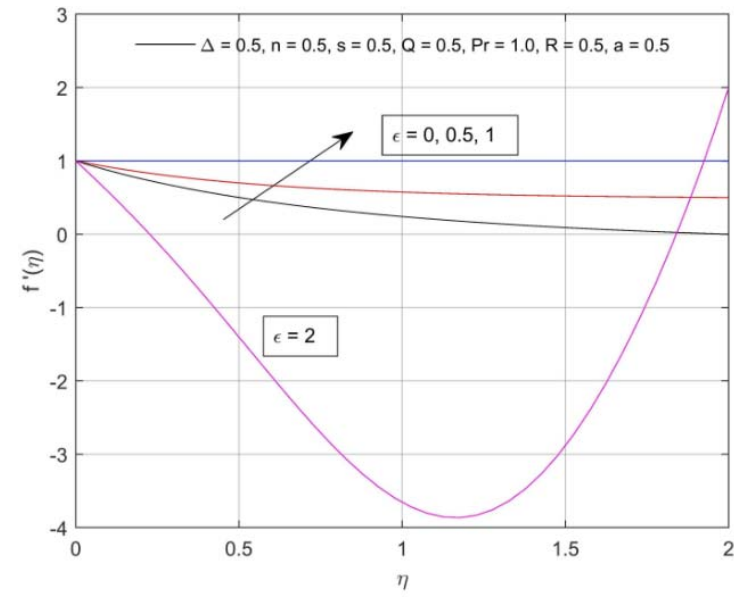

Fig.3. Result for $\epsilon$ with $\mathrm{f}^{\prime}(\eta)$.

From Fig.2. it is apparent that the velocity for the suction case is smaller compared to the injection case. Physically, $s>0$ results in a decrease in the boundary layer thickness and so the velocity reduces. From Fig.3. it is apparent that the velocity increases as $\epsilon$ increases and as long as the potential velocity $\hat{U}_{e} \leq \hat{U}_{w}$. But when $\hat{U}_{e}>\hat{U}_{w}$, the velocity comes down sharply near the elongating wall and then increases. From Fig.4. it can be seen that as $\Delta$ increases the velocity increases and in Fig.5. we observe that the velocity for the stretching sheet is large when compared to the stationary sheet. Also, the parameters $Q$ and $R$ have no impact on the velocity as the equations are not coupled. 


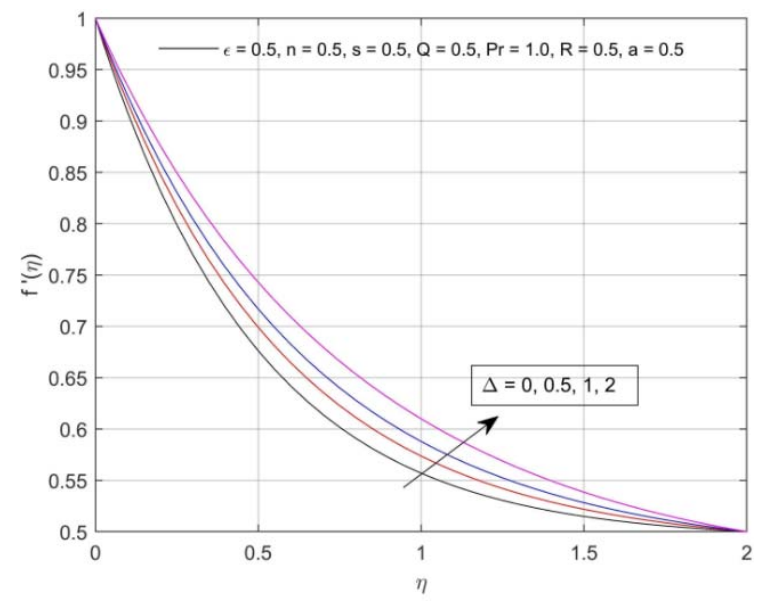

Fig.4. Result for $\Delta$ with $\mathrm{f}^{\prime}(\eta)$.

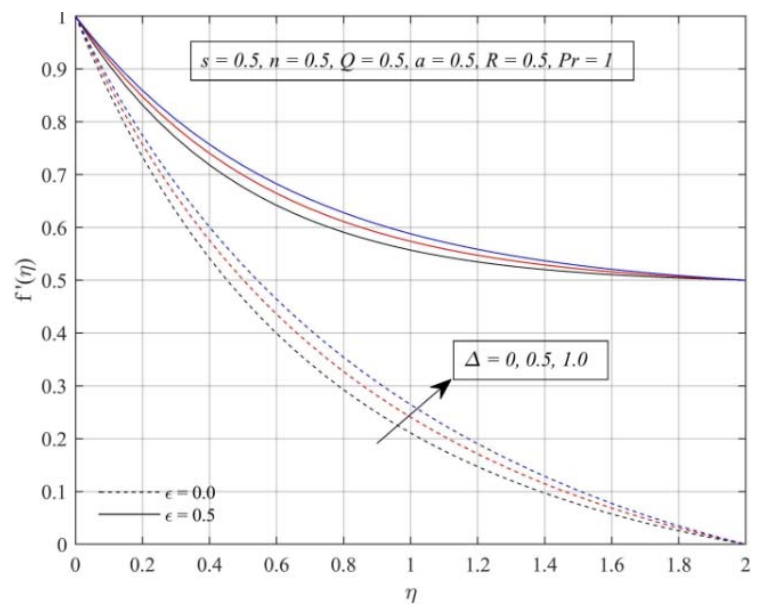

Fig.5. Result for $\Delta$ and $\epsilon$ with $\mathrm{f}^{\prime}(\eta)$.

The impact of $\Delta, \epsilon, s, Q$ and $R$ on the boundary layer temperature trace is depicted in Figs 6-10.

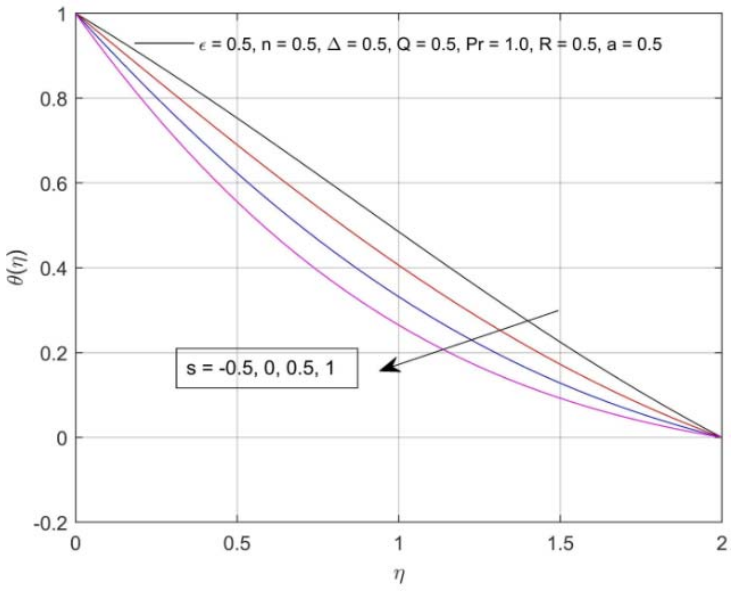

Fig.6. Result for $s$ with $\theta(\eta)$.

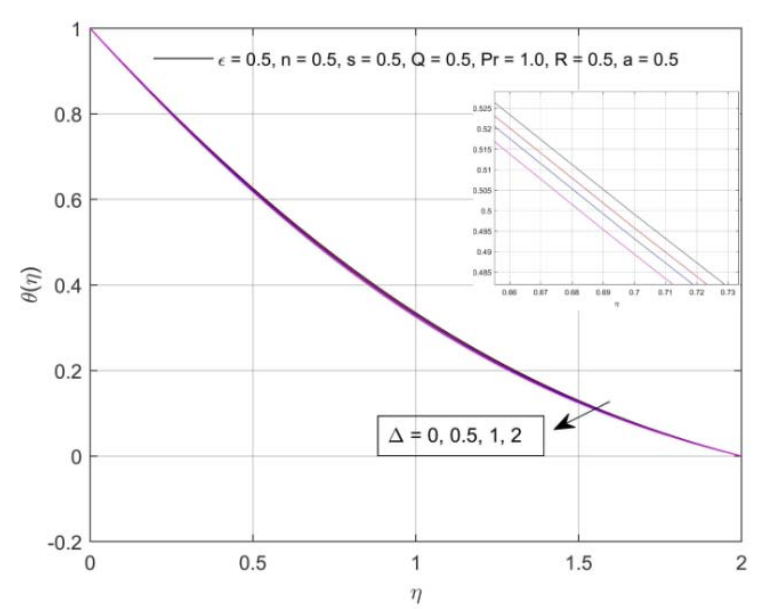

Fig.8. Result for $\Delta$ with $\theta(\eta)$.

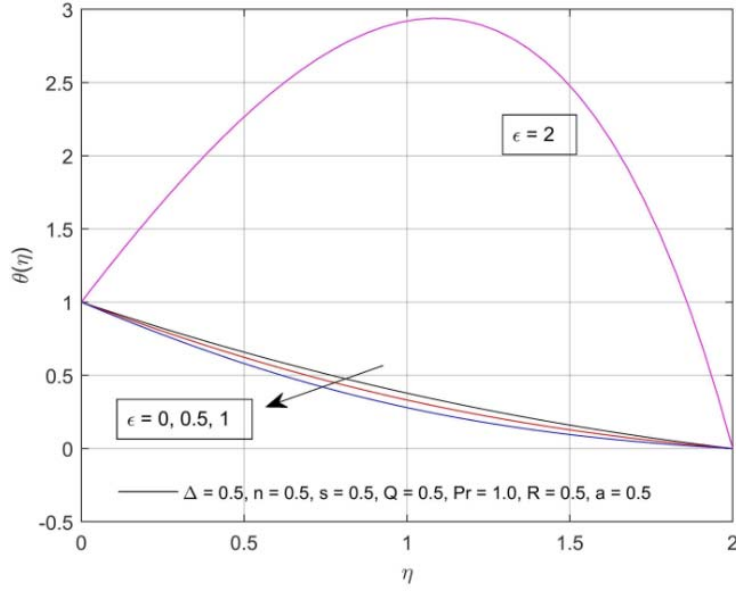

Fig.7. Result for $\epsilon$ with $\theta(\eta)$.

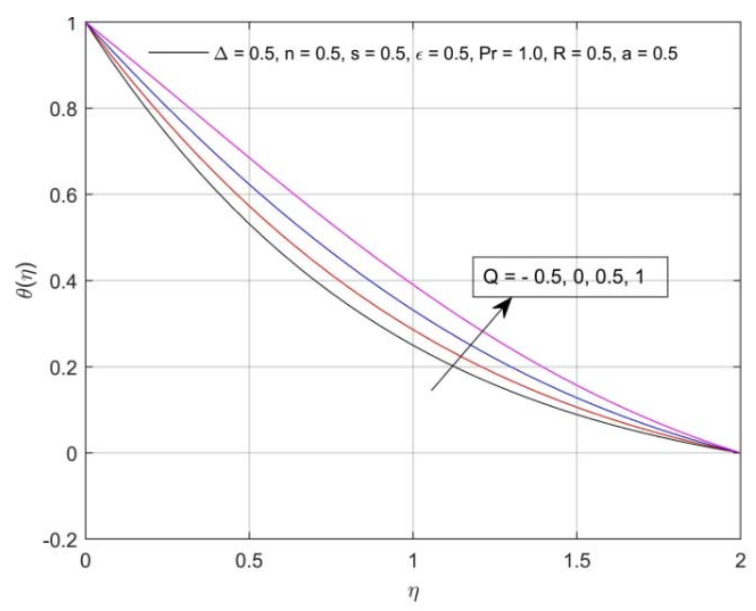

Fig.9. Result for $Q$ with $\theta(\eta)$. 
Figure 6 suggests that the temperature is smaller when $s>0$ (for suction) compared to the case $s<0$ (for blowing). Figure 7 suggests that the temperature drops as $\epsilon$ rises and the potential velocity $\hat{U}_{e} \leq \hat{U}_{w}$. But when $\hat{U}_{e}>\hat{U}_{w}$, the temperature comes up sharply near the elongating wall and then decreases. In Fig.8. we can see that the temperature profile decreases marginally as $\Delta$ increases. Also, Fig.9. suggests that the temperature is high when $Q>0$ (heat source) when compared to the case $Q<0$ (heat sink). As the radiation factor $R$ increases the temperature increases as is evident from Fig.10. Physically, this means that the thermal boundary layer thickness increases with increasing $R$. Also, as the Prandtl number $P r$ increases the temperature decreases as is evident from Fig.11. Physically, this means that the fluids with smaller $P r$ have higher thermal conductivity and larger thermal boundary layer and hence heat diffuses quicker from the sheet.

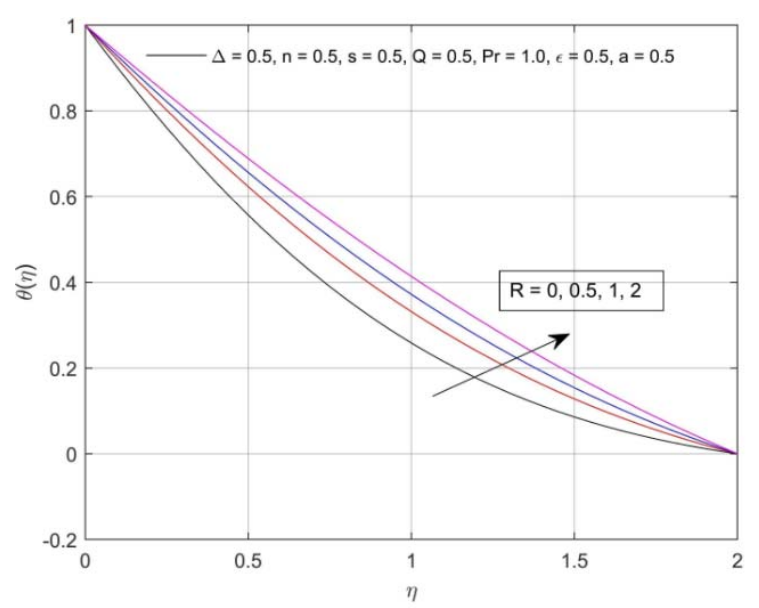

Fig.10. Result for $R$ with $\theta(\eta)$.

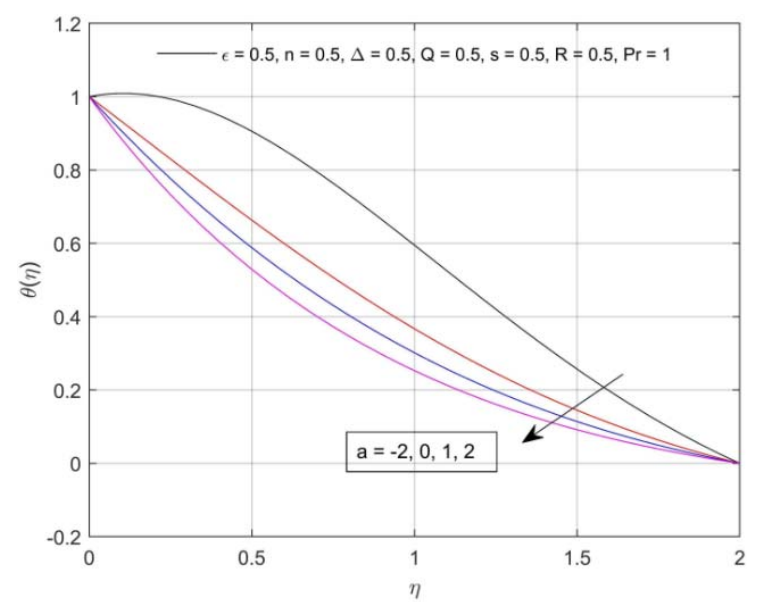

Fig.12. Result for $a$ and $\epsilon=0.5$ with $\theta(\eta)$.

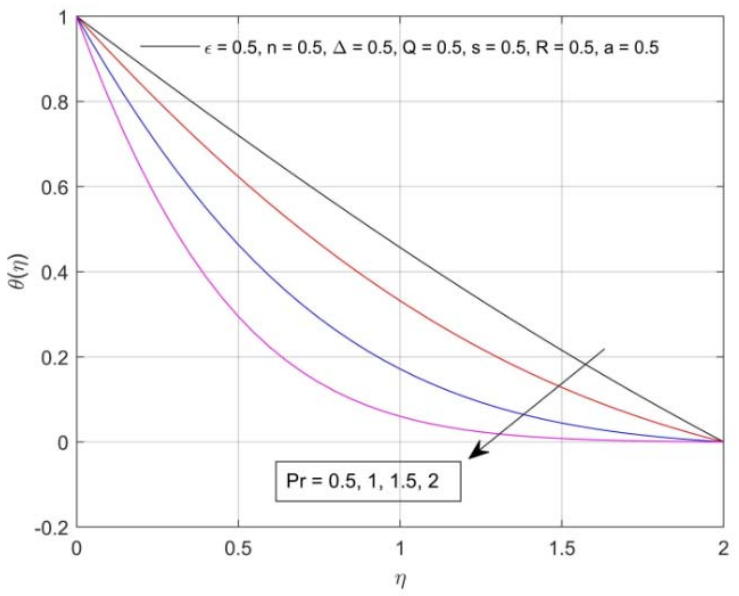

Fig.11. Result for $\operatorname{Pr}$ with $\theta(\eta)$.

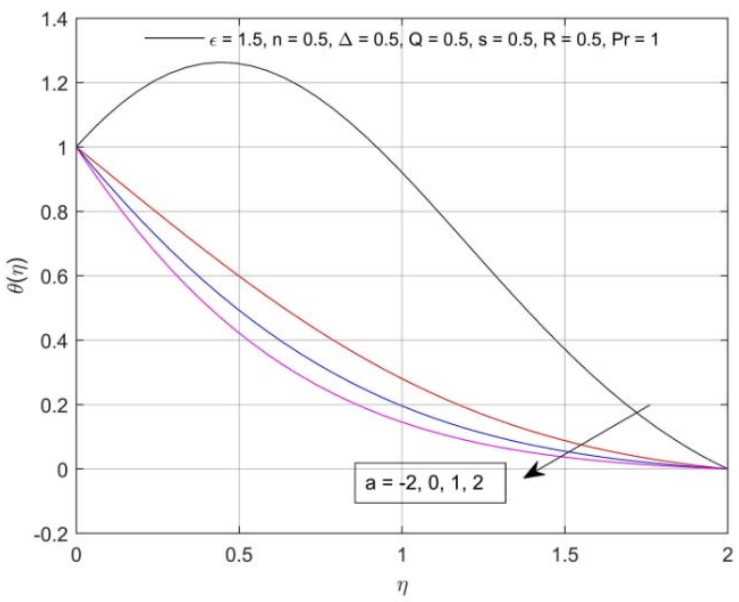

Fig.13. Result for $a$ and $\epsilon=1.5$ with $\theta(\eta)$.

Figures 12 and 13 illustrate the temperature trace in the boundary layer region for different values of the temperature distribution parameter $a$ when $\epsilon=0.5$ and 1.5 and $\operatorname{Pr}=1$. We observe that the temperature decreases as $a$ increases and the temperature increases near the wall of the boundary layer when $a$ is negative but not at the wall. From Figs 14 and 15 we observe that the temperature overshoot when the factor $a$ is negative and both $R, Q$ increase. This is because of the additional heat source and radiation. 


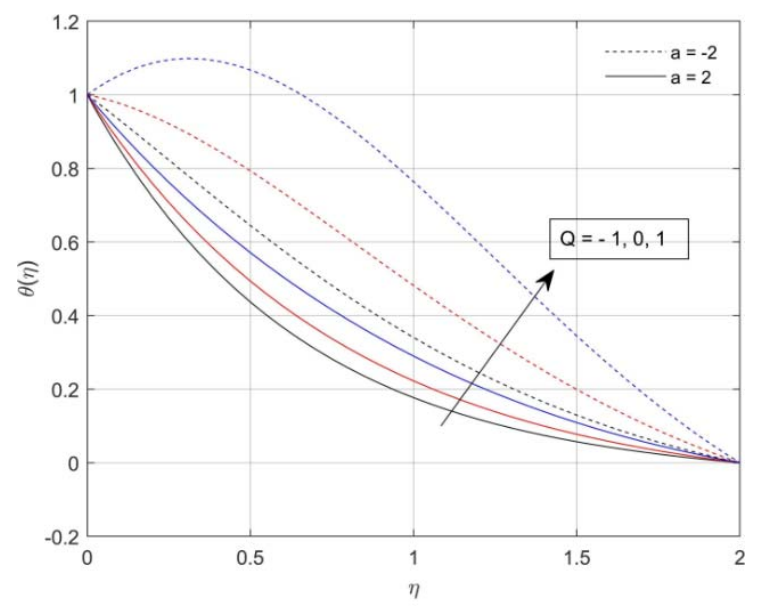

Fig.14. Result for $a$ and $Q$ with $\theta(\eta)$.

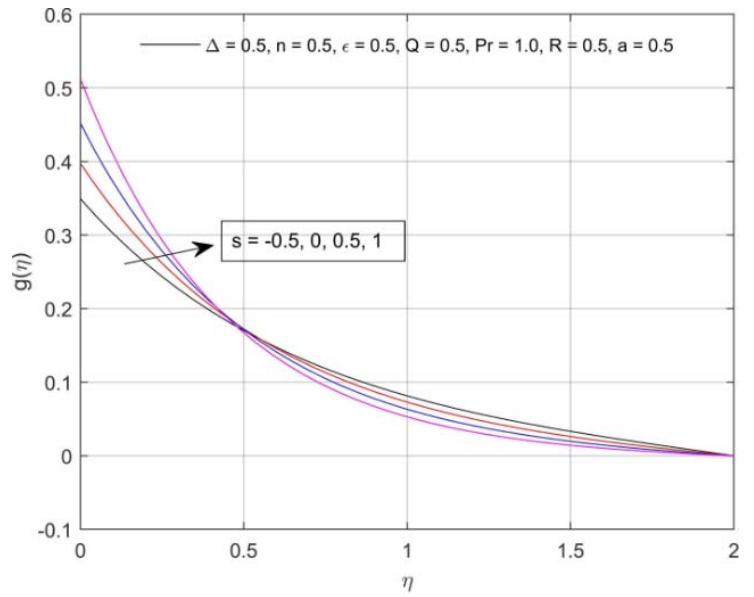

Fig.16. Result for $s$ with $g(\eta)$.

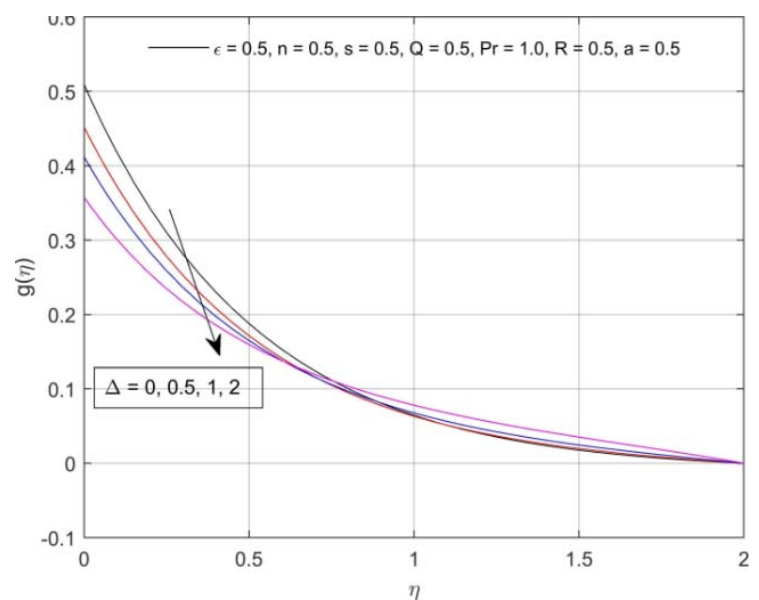

Fig.18. Result for $\Delta$ with $g(\eta)$.

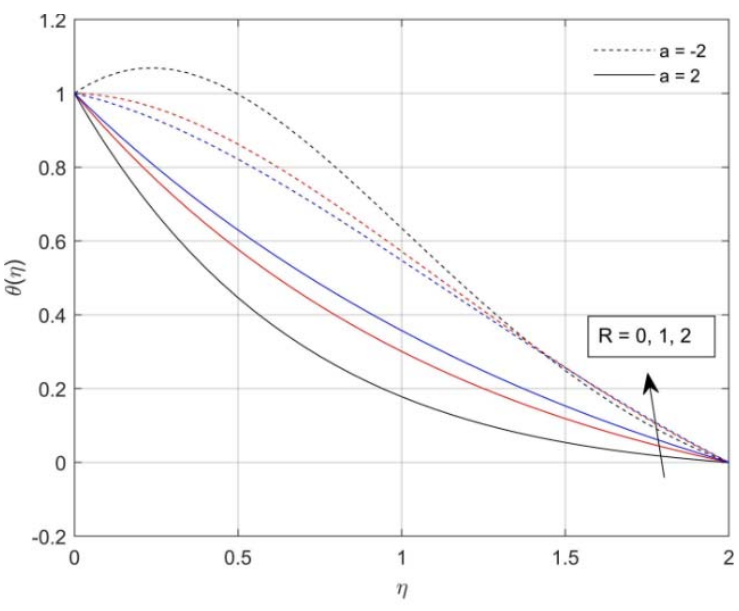

Fig.15. Result for $a$ and $R$ with $\theta(\eta)$.

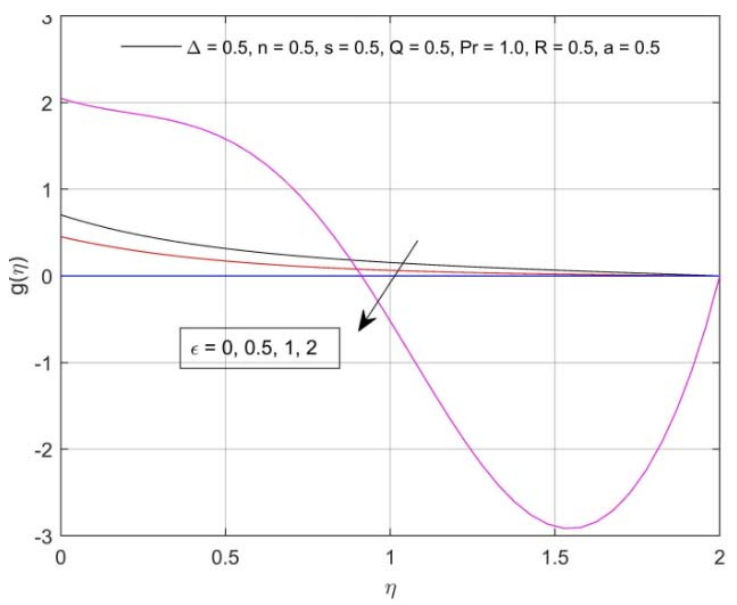

Fig.17. Result for $\epsilon$ with $g(\eta)$.

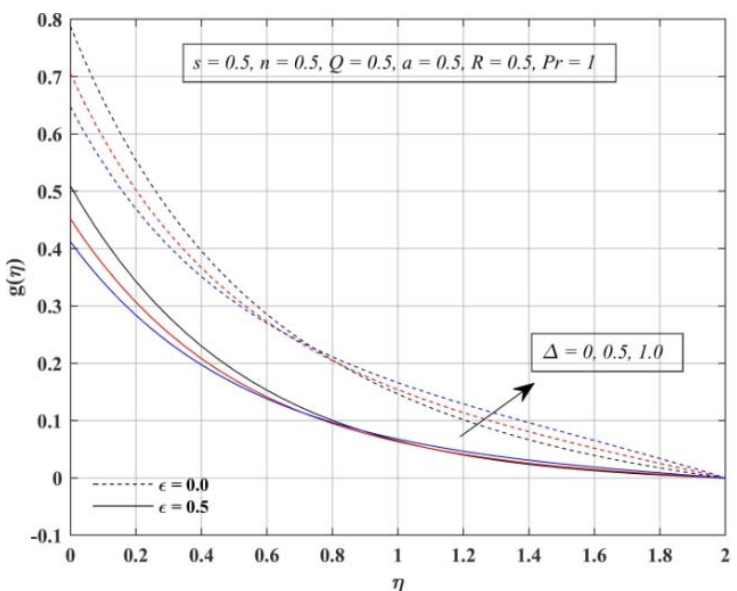

Fig.19. Result for $\Delta$ and $\epsilon$ with $g(\eta)$. 
The impact of $\Delta, \epsilon, s$ on boundary layer angular velocity is depicted in Figs 16-19. From Fig.16. it is apparent that for suction $(s>0)$, the angular velocity is high near the wall when compared to the blowing but the trend reverses as we move away from the wall. Figure 17 suggests that the angular velocity drops as $\epsilon$ rises and the potential velocity $\hat{U}_{e} \leq \hat{U}_{w}$. In Fig.18. we can see that as $\Delta$ increases, the angular velocity shows decreasing trend near the wall but the trend reverses as we move away from the wall. From Fig.19. it is clear that the angular momentum for the stretching sheet is smaller when compared to the static sheet. It is also observed that the parameters $Q$ and $R$ have no impact on the angular velocity as the corresponding equations are not coupled.

Table 1. Variance of $f^{\prime \prime}(0),-\theta^{\prime}(0)$ for combination of parameter values at $n=0.5, a=0.5, \lambda=1$ and $B=1$.

\begin{tabular}{|c|c|c|c|c|c|c|c|c|}
\hline S.No & $\Delta$ & $\epsilon$ & $S$ & $Q$ & $R$ & $\operatorname{Pr}$ & $f^{\prime \prime}(0)$ & $-\theta^{\prime}(0)$ \\
\hline 1 & $\boldsymbol{0}$ & 0.5 & 0.5 & 0.5 & 0.5 & 1 & -1.0183 & 0.8219284 \\
\hline 2 & $\mathbf{0 . 5}$ & 0.5 & 0.5 & 0.5 & 0.5 & 1 & -0.9038941 & 0.8294324 \\
\hline 3 & $\mathbf{1}$ & 0.5 & 0.5 & 0.5 & 0.5 & 1 & -0.8240272 & 0.8352587 \\
\hline 4 & 0.5 & $\boldsymbol{0}$ & 0.5 & 0.5 & 0.5 & 1 & -1.4106 & 0.744 .6304 \\
\hline 5 & 0.5 & $\mathbf{0 . 5}$ & 0.5 & 0.5 & 0.5 & 1 & -0.9038941 & 0.8294324 \\
\hline 6 & 0.5 & $\mathbf{1}$ & 0.5 & 0.5 & 0.5 & 1 & -0.8240272 & 0.8352587 \\
\hline 7 & 0.5 & 0.5 & $\mathbf{- 0 . 5}$ & 0.5 & 0.5 & 1 & -0.6987629 & 0.4592092 \\
\hline 8 & 0.5 & 0.5 & $\boldsymbol{0}$ & 0.5 & 0.5 & 1 & -0.7951157 & 0.6282432 \\
\hline 9 & 0.5 & 0.5 & $\mathbf{0 . 5}$ & 0.5 & 0.5 & 1 & -0.9038941 & 0.8294324 \\
\hline 10 & 0.5 & 0.5 & 0.5 & $\mathbf{- 0 . 5}$ & 0.5 & 1 & -0.9038941 & 1.1746 \\
\hline 11 & 0.5 & 0.5 & 0.5 & $\boldsymbol{0}$ & 0.5 & 1 & -0.9038941 & 1.0133 \\
\hline 12 & 0.5 & 0.5 & 0.5 & $\mathbf{0 . 5}$ & 0.5 & 1 & -0.9038941 & 0.8294324 \\
\hline 13 & 0.5 & 0.5 & 0.5 & 0.5 & $\mathbf{0}$ & 1 & -0.9038941 & 1.0246 \\
\hline 14 & 0.5 & 0.5 & 0.5 & 0.5 & $\mathbf{0 . 5}$ & 1 & -0.9038941 & 0.8294324 \\
\hline 15 & 0.5 & 0.5 & 0.5 & 0.5 & $\mathbf{1}$ & 1 & -0.9038941 & 0.7380408 \\
\hline 16 & 0.5 & 0.5 & 0.5 & 0.5 & 0.5 & $\mathbf{0 . 5}$ & -0.9038941 & 0.5724855 \\
\hline 17 & 0.5 & 0.5 & 0.5 & 0.5 & 0.5 & $\mathbf{1}$ & -0.9038941 & 0.8294324 \\
\hline 18 & 0.5 & 0.5 & 0.5 & 0.5 & 0.5 & $\mathbf{2}$ & -0.9038941 & 2.0683 \\
\hline
\end{tabular}

\section{Conclusions}

The impact of $\Delta, \epsilon, s, Q, R$ and $\operatorname{Pr}$ on $f^{\prime}(0), g(0)$ and $\theta(0)$ are as observed:

1. As long as $\epsilon$ increases and the potential velocity $\hat{U}_{e} \leq \hat{U}_{w}$ the elongating wall velocity, the temperature and angular velocity decrease but the velocity increases. When $\hat{U}_{e}>\hat{U}_{w}$, the temperature and angular velocity come down sharply near the stretching wall and then increase but a reverse trend is seen for the velocity.

2. The velocity and temperature for suction $(s>0)$ is smaller when compared to the injection $(s<0)$. The angular velocity is high near the wall for suction when compared to the blowing but the trend reverses as we move away from the wall. 
3. As the micropolar factor $\Delta$ surges the velocity trace surges but the temperature profile decreases marginally. As $\Delta$ increases the angular velocity shows a decreasing trend near the wall but the trend reverses as we move away from the wall.

4. The factors $Q$ and $R$ have no impact on the velocity and the angular velocity. The temperature surges as $Q$ and $R$ rise whereas the Prandtl number increases with a decrease in temperature.

\section{Nomenclature:}

$$
\begin{aligned}
a & - \text { temperature distribution parameter } \\
C_{p} & - \text { specific heat at constant pressure } \\
C_{f} & \text { - shearing stress } \\
f^{\prime} & - \text { dimensionless fluid velocity } \\
g^{\prime} & - \text { dimensionless angular velocity } \\
j & - \text { microinertia density } \\
l & - \text { reference length } \\
L & - \text { length } \\
n & - \text { boundary value factor } \\
\hat{N} & - \text { microrotation } \\
N u_{\hat{x}} & - \text { Nusselt number } \\
P r & - \text { Prandtl number } \\
Q & - \text { heat source/sink factor } \\
q_{w} & - \text { surface heat flux } \\
R e_{\hat{x}} & - \text { local Reynolds number } \\
s & - \text { suction/injection factor } \\
\hat{T} & - \text { fluid temperature } \\
\hat{T}_{\infty} & - \text { free stream temperature } \\
\hat{u} & - \text { velocity along the surface } \\
\hat{u}_{e} & - \text { free stream velocity } \\
\hat{U}_{I} & - \text { wall stretching factor } \\
\hat{U}_{0} & - \text { free stream velocity factor } \\
\hat{v} & - \text { velocity perpendicular to the surface } \\
\hat{v}_{w}(\hat{x}) & - \text { transpiration velocity } \\
\hat{x} & - \text { direction along the surface } \\
\hat{y} & - \text { direction perpendicular to the surface } \\
\gamma & - \text { spiral gradient } \\
\delta & - \text { slip factor } \\
\Delta & - \text { micropolar factor } \\
\varepsilon & - \text { shrinking factor } \\
\eta & - \text { boundary layer length } \\
\theta & - \text { dimensionless temperature } \\
v & - \text { kinematic viscosity } \\
&
\end{aligned}
$$




$$
\begin{array}{cl}
\kappa & - \text { vortex viscosity } \\
\mu & - \text { dynamic viscosity } \\
\rho & - \text { fluid density } \\
\tau_{w} & - \text { surface shear stress }
\end{array}
$$

\section{References}

[1] Eringen A.C. (1966): Theory of micropolar fluids.- J. Math. Mech., vol.16, pp.1-18.

[2] Mukhopadhyay S. (2013): Slip effects on MHD boundary layer flow over an exponentially stretching sheet with suction/blowing and thermal radiation.- Ain Shams Eng. J., vol.4, pp.485-491.

[3] Mukhopadhyay S., Layek G.C. and Gorla R.S.R. (2007): MHD combined convective flow and heat transfer past a porous stretching surface.- Int. J. Fluid Mech. Res., vol.34, pp.244-257.

[4] Mahapatra Ray T., Dholey S. and Gupta A.S. (2007): Oblique stagnation-point flow of an incompressible visco-elastic fluid towards a stretching surface.- Int. J. Non-Linear Mech., vol.42, pp.484-499.

[5] Mukhopadhyay S. and Gorla R.S.R. (2012): Effects of partial slip on boundary layer flow past a permeable exponential stretching sheet in presence of thermal radiation.- Heat Mass Transfer, vol.48, pp.1773-1781.

[6] Crane L.J. (1970): Flow past a stretching plate.- Z Angew Math Phys., vol.21, pp.64-67.

[7] Carragher P. and Crane L.J. (1982): Heat transfer on a continuous stretching sheet.- Z. Angew. Math. Mech., vol.62, p.564.

[8] Gupta P.S. and Gupta A.S. (1977): Heat and mass transfer on a stretching sheet with suction or blowing.- Can. J. Chem. Eng., vol.55, pp.744-746.

[9] Chakrabarti A., Gupta A.S. (1979): Hydromagnetic flow and heat transfer over a stretching sheet.- Quart. Appl. Math., vol.33, pp.73-78.

[10] Hady F.M. (1996): Short communication on the solution of heat transfer to micropolar fluid from a non-isothermal stretching sheet with injection.- Int. J. Num. Meth. Heat Fluid Flow, vol.6, pp.99-104.

[11] Hassanien I.A. and Gorla R.S.R. (1990): Heat transfer to a micropolar fluid from a non isothermal stretching sheet with suction and blowing.- Acta Mech., vol.84, pp.191-199.

[12] Hayat T., Abbas Z. and Javed T. (2008): Mixed convection flow of a micropolar fluid over a non- linear stretching sheet.- Phys. Lett. A., vol.372, pp.637-647.

[13] Chen C.K. and Char M.I. (1988): Heat transfer of a continuous stretching surface with suction or blowing.- J. Math. Anal. Appl., vol.135, pp.568-580.

[14] Datta B.K., Roy P. and Gupta A.S. (1985): Temperature field in the flow over a stretching sheet with uniform heat flux.- Int. Comm. Heat Mass., Tran. 12, 89-94.

[15] Vajravelu K. (1994): Convection heat transfer at a stretching sheet with suction or blowing.- J. Math. Anal. Appl., vol.188, No.3, pp.1002-1011.

[16] Mukhopadhyay S., Mandal I.C. and Gorla R.S.R . (2012): Effects of thermal stratification on flow and heat transfer past a porous vertical stretching surface.- Heat Mass Transfer, vol.48, pp.915-921.

[17] Ishak A., Nazar R. and Pop I. (2008): Hydromagnetic flow and heat transfer adjacent to a stretching vertical sheet.Heat Mass Transfer., vol.44, pp.921-927.

[18] Van Gorder R.A. and Vajravelu K. (2009): A note on flow geometries and the similarity solutions of the boundary layer equations for a nonlinearly stretching sheet.- Arch. Appl. Mech. vol.80, pp.1329-1332.

[19] Mukhopadhyay S. (2013): Analysis of boundary layer flow over a porous nonlinearly stretching sheet with partial slip at the boundary.- Alex Eng. J., vol.52, pp.563-569. 
[20] Vajravelu K. and Rollins D. (1992): Heat transfer in electrically conducting fluid over a stretching sheet.- Int. J. Non-linear Mech., vol.27, pp.265-277.

[21] Mahmoud M.A.A. and Waheed S.E. (2012): MHD flow and heat transfer of a micropolar fluid over a stretching surface with heat generation (absorption) and slip velocity.- Journal of the Egyptian Mathematical Society., vol.20, pp.20-27.

[22] Magyari E. and Keller B. (1999): Heat and mass transfer in the boundary layers on an exponentially stretching continuous surface.- J. Phys. D Appl. Phys., vol.32, pp.577-585.

[23] Elbashbeshy E.M.A. (2001): Heat transfer over an exponentially stretching continuous surface with suction.- Arch. Mech., vol.53, pp.643-651.

[24] Sajid M. and Hayat T. (2008): Influence of thermal radiation on the boundary layer flow due to an exponentially stretching sheet.- Int. Comm. Heat Mass Tran., vol.35, pp.347-356.

[25] Bidin B. and Nazar R. (2009): Numerical solution of the boundary layer flow over an exponentially stretching sheet with thermal radiation.- Eur. J. Sci. Res., vol.33, No.4, pp.710-717.

[26] Pal D. (2010): Mixed convection heat transfer in the boundary layers on an exponentially stretching surface with magnetic field.- Appl. Math. Comput., vol.217, pp.2356-2369.

[27] Ishak A. (2011): MHD boundary layer flow due to an exponentially stretching sheet with radiation effect.- Sains Malaysiana., vol.40, pp.391-395.

[28] Schlichting H. (1960): Boundary Layer Theory.- McGraw-Hill.

[29] Goldstein S. (1938): Modern Development in Fluid Dynamics.- Oxford University Press, London.

[30] Maiti M.K. (1965): Axially-symmetric stagnation point flow of power law fluids.- Z. Angew. Math. Phys., vol.16, pp.594-598.

[31] Koneru S.R. and Manohar R. (1968): Stagnation point flows of non-Newtonian power law fluids.- Z. Angew. Math. Phys., vol.19, pp.84-88.

[32] Mahapatra T.R and Gupta A.S. (2002): Heat transfer in stagnation-point flow towards a stretching sheet.- Heat Mass Transfer., vol.38, pp.517-521.

[33] Stuart J.T. (1959): The viscous flow near a stagnation point when the external flow has uniform vorticity.- J. Aerospace Sci., vol.26, pp.124-125.

[34] Nazar R., Amin N., Filip D. and Pop I. (2004): Stagnation point flow of a micropolar fluid towards a stretching sheet.- Int. J. Non-Linear Mech., vol.39, pp.1227-35.

[35] Kai-Long Hsiao (2010): Heat and mass transfer for micropolar flow with radiation effect past a nonlinearly stretching sheet.- Heat Mass Transfer., vol.46, pp.413-419.

[36] Alavi S.Q., Abid Hussanan A., Kasim A.R.M., Rosli N. and Salleh M.Z. (2017): MHD stagnation point flow towards an exponentially stretching sheet with prescribed wall temperature and heat flux.- Int. J. Appl. Comput. Math., vol.3, pp3511-3523.

[37] Mukhopadhyay S. (2013): Slip effects on MHD boundary layer flow over an exponentially stretching sheet with suction/blowing and thermal radiation.- Ain Shams Eng. J., vol.4, No.3, pp.485-491

[38] Chaudhary S., Singh S. and Chaudhary S. (2015): Thermal radiation effects on MHD boundary layer flow over an exponentially stretching surface.- Applied Mathematics., vol.6, pp.295-303.

Received: July 6, 2021

Revised: October 5, 2021 